\title{
Resource Portfolio Problem under Relaxed Resource Dedication Policy in Multi-mode Multi-project Scheduling
}

Umut Beşikçi

Triodor ArGe Yazılım ve Bilişim Tic. Ltd. Şti., Aydınevler, Durak Sokak 7/9, Maltepe, 34854 Istanbul, Turkey

Email: umut.besikci@triodor.eu

Ümit Bilge*

Department of Industrial Engineering, Boğaziçi University, Bebek, 34342 Istanbul, Turkey

Email: bilge@boun.edu.tr

*Corresponding author

Gündüz Ulusoy

Faculty of Engineering and Natural Sciences,

Sabancı University, Orhanl, Tuzla, 34956 Istanbul, Turkey

Email:gunduz@sabanciuniv.edu

Abstract: The most common approach in the multi-project scheduling literature considers resources as a common pool shared among all projects. However, different resource management strategies may be required for different problem environments. We present the Relaxed Resource Dedication (RRD) policy, which prevents the sharing of resources among projects but allows resource transfers when a project starts after the completion of another one. We treat the case where the available amounts of resources -namely, the capacities- are decision variables subject to a limited budget. This capacity planning problem, called the Resource Portfolio Problem, is investigated under the RRD policy employing both renewable and nonrenewable resources with multiple modes of usage. A mixed integer linear programming model to minimize total weighted tardiness is proposed. To obtain some benchmark solutions for this hard problem, the branch and cut procedure of ILOG CPLEX is modified by customized branching strategies, feasible solution generation schemes and valid inequalities.

Keywords: Multi-mode Resource Constrained Multi-project Scheduling; Resource Dedication; Resource Portfolio Allocation; Branch and Cut.

Appeared in International Journal of Mathematics in Operational Research (IJMOR), 14(4), 541-567, 2019. 